Rapporto n. 227

Isotonicity properties of generalized quantiles

\author{
Fabio BEШNI
}

Marzo 2012

Dipartimento di Metodi Quantitativi per le Scienze Economiche ed Aziendali Università degli Studi di Milano Bicocca

Via Bicocca degli Arcimboldi 8 - 20126 Milano - Italia

Tel +39/02/64483102/3- Fax +39/2/64483105

Segreteria di redazione: Andrea Bertolini 


\title{
Isotonicity properties of generalized quantiles
}

\author{
Fabio Bellini*
}

March 22, 2012

\begin{abstract}
We investigate whether several families of generalized quantiles (expectiles, $L^{p}$-quantiles and $M$-quantiles) respect various stochastic orders (the usual stochastic order, the convexity order, the $s$-convexity orders). We employ techniques from monotone comparative statics developed in Topkis (1978) and Milgrom and Shannon (1994), in order to provide sufficient as well as necessary conditions for isotonicity.

We show that expectiles with $\alpha<1 / 2$ are basically the only generalized quantiles that are isotonic with respect to the $\leq_{i c v}$ ordering; more generally, the $L^{p}$-quantiles are isotonic with respect to the $s$-convex order.
\end{abstract}

Keywords: expectiles, generalized quantiles, stochastic orders, isotonicity, submodularity, single crossing condition

\section{Introduction}

It is well known that the quantiles of a distribution can be defined as the minimizers of an asymmetric piecewise linear loss function:

$$
x_{\alpha}^{*}(X)=\min _{x \in R} \pi_{\alpha}(X, x)
$$

with

$$
\pi_{\alpha}(X, x):=E\left[\alpha(X-x)^{+}+(1-\alpha)(X-x)^{-}\right] .
$$

This very important property has been exploited in various areas of statistics. It has been introduced in the context of statistical decision theory (see Ferguson (1967)); it lies at the heart of the quantile regression approach developed by Koenker and Bassett (see Koenker (2005)); more recently, it has been employed in financial risk management in Rockafellar and Uryasev (2002).

Alternative specifications of the loss function (2) have originated several kind of generalized quantiles: Newey and Powell (1987) considered a quadratic loss

*Dipartimento di Metodi Quantitativi, Università di Milano Bicocca, Italy. E-mail: fabio.bellini@unimib.it 
(expectiles), Chen (1996) a $p$-power loss ( $L^{p}$-quantiles), Breckling and Chambers (1988) generic convex losses ( $M$-quantiles). Similar functionals have also been considered in the actuarial literature under the name of Orlicz quantiles in Bellini and Rosazza Gianin (2011).

When considering the usual quantiles, it is well known that if $X \leq_{s t} Y$, then $x_{\alpha}^{*}(X) \leq x_{\alpha}^{*}(Y)$; that is, quantiles are isotonic with respect to the usual stochastic order $\leq_{s t}$.

In this paper we investigate similar isotonicity properties for generalized quantiles; this is relevant in that provides additional information on their significance and possible interpretation.

For example, we will show that expectiles (with $\alpha \leq \frac{1}{2}$ ) are isotone with respect to the $\leq_{i c v}$ ordering, also known as second order stochastic dominance in the financial literature. This property shows that expectiles might be reasonable risk measures for a risk averse agent, as it was suggested also in Muller (2010). Moreover, we will show that the expectiles are basically the only $M$-quantiles that share this isotonicity property.

To prove these results, we employ techniques from monotone comparative statics, developed in the seminal papers of Topkis (1978) and Milgrom and Shannon (1994). Roughly, this theory provides sufficient and necessary condition for increasing optimal solutions in a parametric optimization problem as (1) (the variable $X$ is seen as a parameter), under suitable conditions on the loss function $\pi_{\alpha}(X, x)$.

\section{Expectiles}

The expectiles have been introduced by Newey and Powell (1987) as the minimizers

$$
x_{\alpha, X}^{*}:=\arg \min _{x \in R} \pi_{\alpha}(X, x)
$$

of the asymmetric quadratic loss

$$
\pi_{\alpha}(X, x)=E\left[\alpha\left((X-x)^{+}\right)^{2}+(1-\alpha)\left((X-x)^{-}\right)^{2}\right]
$$

with $\alpha \in(0,1)$ and $E\left[|X|^{2}\right]<+\infty$. In order to avoid unnecessarily heavy notations, we will write simply $x^{*}$ or $x_{\alpha}^{*}$ when no possibility of confusion arises. Clearly if $\alpha=\frac{1}{2}$ then

$$
\pi_{\frac{1}{2}}(X, x)=\frac{1}{2} E\left[(X-x)^{2}\right]
$$

so that

$$
x_{\frac{1}{2}, X}^{*}=E[X]
$$

Newey and Powell proved that $x^{*}$ is always unique and satisfies the first order 
condition

$$
x^{*}-E[X]=\frac{2 \alpha-1}{1-\alpha} \int_{x^{*}}^{+\infty}\left(t-x^{*}\right) d F(t)
$$

that can be rewritten in the equivalent forms

$$
(1-\alpha) \int_{-\infty}^{x^{*}} F(t) d t=\alpha \int_{x^{*}}^{+\infty} \bar{F}(t) d t
$$

or

$$
\alpha=\frac{E\left[\left(X-x^{*}\right)^{-}\right]}{E\left[\left|X-x^{*}\right|\right]}
$$

with $\bar{F}(t)=1-F(t)$. These equations are well defined and have a unique solution also for $X \in L^{1}$. The basic properties of expectiles are summarized in the following proposition:

Proposition 1 (Nevey and Powell, 1987) Let $E[|X|]<+\infty, \alpha \in(0,1), x_{\alpha, X}^{*}$ as in (7). It follows that:

- $x_{\alpha, X}^{*}$ is strictly monotonic with respect to $\alpha$

- $x_{\alpha, X}^{*}$ is positively homogeneous: $x_{\alpha, \lambda X}^{*}=\lambda x_{\alpha, X}^{*}, \forall \lambda \geq 0$

- $x_{\alpha, X}^{*}$ is translation equivariant: $x_{\alpha, X+c}^{*}=x_{\alpha, X}^{*}+c, \forall c \in R$

- If $X$ is symmetric with respect to $x_{0}$, then

$$
\frac{x_{\alpha, X}^{*}+x_{1-\alpha, X}^{*}}{2}=x_{0}
$$

- If $X$ has a continuous density, then $x_{\alpha, X}^{*}$ is $C^{1}$ as a function of $\alpha$, with

$$
\frac{d x_{\alpha}^{*}}{d \alpha}=\frac{E\left[\left|X-x^{*}\right|\right]}{(1-\alpha) F\left(x^{*}\right)+\alpha \bar{F}\left(x^{*}\right)}
$$

Typically expectiles are more concentrated around the mean than the corresponding quantiles. However, a general comparison result is still lacking and Koenker (1992) showed that for the infinite variance distribution function given by

$$
F(t)=\left\{\begin{array}{l}
\frac{1}{2}\left(1+\sqrt{1-\frac{4}{4+t^{2}}}\right), t \geq 0 \\
\frac{1}{2}\left(1-\sqrt{1-\frac{4}{4+t^{2}}}\right), t<0
\end{array}\right.
$$

expectiles coincide with quantiles.

Jones (1994) remarked that

$$
G(x):=\frac{E\left[\left(X-x^{*}\right)^{-}\right]}{E\left[\left|X-x^{*}\right|\right]}
$$


is actually a distribution function, so from equation (9) it follows that the expectiles of $F$ are the quantiles of the new distribution $G$.

Rewriting equation (7) we find also that

$$
x^{*}=\frac{\alpha E\left[X 1_{\left\{X>x^{*}\right\}}\right]+(1-\alpha) E\left[X 1_{\left\{X \leq x^{*}\right\}}\right]}{\left.\alpha P\left(X>x^{*}\right)+(1-\alpha) P\left(X \leq x^{*}\right)\right]}=E\left[X \varphi\left(X, x^{*}\right)\right]
$$

with

$$
\varphi\left(X, x^{*}\right):=\frac{\alpha 1_{\left\{X>x^{*}\right\}}+(1-\alpha) 1_{\left\{X \leq x^{*}\right\}}}{E\left[\alpha 1_{\left\{X>x^{*}\right\}}+(1-\alpha) 1_{\left\{X \leq x^{*}\right\}}\right]}
$$

that shows that expectiles can also be seen as averages with respect to a new measure whose Radon-Nikodym density $\varphi$ is specified by (15). This leads to the following simple formula in the discrete case:

Example 2 If $X$ has a discrete equiprobable distribution with values $x_{1}<x_{2}<$ $\ldots<x_{n}$, defining

$$
\alpha_{i}^{*}:=\frac{i x_{i}-\sum_{k=1}^{i} x_{k}}{\sum_{k=1}^{n}\left|x_{k}-x_{i}\right|}, i=1, \ldots, n
$$

then for $\alpha \in\left(\alpha_{i}^{*}, \alpha_{i+1}^{*}\right)$ the expectile $x_{\alpha, X}^{*}$ is given by

$$
x_{\alpha, X}^{*}=\frac{(1-\alpha) x_{1}+\ldots(1-\alpha) x_{i}+\alpha x_{i+1}+\ldots+\alpha x_{n}}{i+\alpha(n-2 i)} .
$$

\subsection{Isotonicity results}

The aim of this section is to establish the isotonicity of expectiles with respect to the first and second order stochastic dominances (also known respectively as usual stochastic order and increasing concave order in the statistical literature). We recall the standard definitions (see for example Muller and Stoyan, 2002):

- $X \leq_{s t} Y$ if and only if $E[f(X)] \leq E[f(X)]$ for each increasing $f$,

- $X \leq_{c v} Y$ if and only if $E[f(X)] \leq E[f(X)]$ for each concave $f$,

- $X \leq_{i c v} Y$ if and only if $E[f(X)] \leq E[f(X)]$ for each increasing and concave $f$, where in all cases both expected values must be well defined.

We pursue a purely order-theoretic comparative static approach, in the spirit of Topkis (1978) and Milgrom and Shannon (1994); a recent reference book on the subject is Topkis (1998). In the defining minimization problem

$$
x_{\alpha, X}^{*}=\arg \min _{x \in R} \pi_{\alpha}(X, x)
$$


we consider $X$ as a parameter, belonging to the partially ordered set $\left(L^{2}, \precsim\right.$ ), where the role of the partial order $\precsim$ will be played in turn by $\leq_{s t}, \leq_{c v}$ and $\leq_{i c v}$. The theory of monotone comparative statics provides necessary and sufficient conditions on the function $\pi_{\alpha}(X, x)$ for increasing optimal solutions, that is in order to have that $X \precsim Y \Longrightarrow x_{\alpha, X}^{*} \leq x_{\alpha, Y}^{*}$.

With respect to the general theory, our problem is particularly simple in three aspects:

i) the minimizer is always unique

ii) the domain of the minimization problem (16) does not depend on the parameter $X$

iii) the decision variable $x$ belongs to the totally ordered set $R$.

Under these hypothesis, a sufficient condition for increasing optimal solutions is that $\pi_{\alpha}(x, X)$ has antitone differences (see Theorem 2.8.1 in Topkis (1998)), that is

$$
\pi_{\alpha}(X, y)-\pi_{\alpha}(X, x) \geq \pi_{\alpha}(Y, y)-\pi_{\alpha}(Y, x)
$$

for each $x \leq y$ and $X \preceq Y$.

An equivalent way of writing (17) is

$$
\pi_{\alpha}(X, y)+\pi_{\alpha}(Y, x) \geq \pi_{\alpha}(Y, y)+\pi_{\alpha}(X, x)
$$

that is equivalent to the submodularity of the function $\pi_{\alpha}(X, y)$ on the product lattice $L^{2} \times R$.

Theorem 3 Let $x_{\alpha, X}^{*}$ as in (16). We have the following:

a) If $X \leq_{s t} Y$, then $x_{\alpha, X}^{*} \leq x_{\alpha, Y}^{*}$ for each $\alpha \in(0,1)$;

b) If $X \leq_{c v} Y$, then $x_{\alpha, X}^{*} \leq x_{\alpha, Y}^{*}$ for each $\alpha<\frac{1}{2}$ and $x_{\alpha, X}^{*} \geq x_{\alpha, Y}^{*}$ for each $\alpha>\frac{1}{2}$

c) If $X \leq_{i c v} Y$, then $x_{\alpha, X}^{*} \leq x_{\alpha, Y}^{*}$ for each $\alpha<\frac{1}{2}$.

Proof. We start by computing $\pi_{\alpha}(X, y)-\pi_{\alpha}(X, x)$. Since $x<y$, we have

$$
\begin{aligned}
& \left((X-y)^{-}\right)^{2}=(y-X)^{2} 1_{\{X \leq x\}}+(y-X)^{2} 1_{\{x \leq X \leq y\}} \\
& \left((X-x)^{+}\right)^{2}=(X-x)^{2} 1_{\{X>y\}}+(X-x)^{2} 1_{\{x \leq X \leq y\}}
\end{aligned}
$$

that gives

$$
\begin{aligned}
& \pi_{\alpha}(X, y)-\pi_{\alpha}(X, x)= \\
& E\left[\alpha\left((X-y)^{+}\right)^{2}+(1-\alpha)\left((X-y)^{-}\right)^{2}\right]-E\left[\alpha\left((X-x)^{+}\right)^{2}+(1-\alpha)\left((X-x)^{-}\right)^{2}\right]= \\
& E\left[\alpha(X-y)^{2} 1_{\{X>y\}}+(1-\alpha)\left\{(y-X)^{2} 1_{\{X \leq x\}}+(y-X)^{2} 1_{\{x \leq X \leq y\}}\right\}\right]- \\
& -E\left[\alpha\left\{(X-x)^{2} 1_{\{X>y\}}+(X-x)^{2} 1_{\{x \leq X \leq y\}}\right\}+(1-\alpha)(x-X)^{2} 1_{\{X \leq x\}}\right]= \\
& E\left[(1-\alpha) 1_{\{X \leq x\}}\left\{(y-X)^{2}-(x-X)^{2}\right\}\right] \\
& +E\left[\alpha 1_{\{X>y\}}\left\{(X-y)^{2}-(X-x)^{2}\right\}\right] \\
& +E\left[1_{\{x \leq X \leq y\}}\left\{(1-\alpha)(y-X)^{2}-\alpha(X-x)^{2}\right\}\right]:=E\left[g_{x, y}(X)\right]
\end{aligned}
$$


with

$$
g_{x, y}(t):=\left\{\begin{array}{c}
(1-\alpha)\left(2(x-y) t+y^{2}-x^{2}\right) \text { if } t \leq x \\
\left.(1-\alpha)(y-t)^{2}-\alpha(t-x)^{2}\right) \text { if } x<t \leq y \\
\alpha\left(2(x-y) t+y^{2}-x^{2}\right) \text { if } t>y
\end{array}\right.
$$

As we already recalled, from Theorem 2.8.1 in Topkis (1998) the submodularity of $\pi_{\alpha}(X, x)$ is a sufficient condition for increasing optimal solutions. From the preceding computation, we have that

$$
\pi_{\alpha}(X, y)-\pi_{\alpha}(X, x)-\pi_{\alpha}(Y, y)+\pi_{\alpha}(Y, x)=E\left[g_{x, y}(X)\right]-E\left[g_{x, y}(Y)\right]
$$

In order to prove a), we note that $g_{x, y}(t)$ is decreasing for each $\alpha \in(0,1)$, and since $X \leq_{s t} Y$ we have that $E\left[g_{x, y}(X)\right] \geq E\left[g_{x, y}(Y)\right]$, that gives condition (17). It follows that $x_{\alpha, X}^{*} \leq x_{\alpha, Y}^{*}$ for each $\alpha \in(0,1)$.

Similarly, we note that when $\alpha \in\left(0, \frac{1}{2}\right)$, the function $g_{x, y}(t)$ is convex; it follows that if $X \leq_{c v} Y$, then $E\left[g_{x, y}(X)\right]-E\left[g_{x, y}(Y)\right] \geq 0$, that gives condition (17) and $x_{\alpha, X}^{*} \leq x_{\alpha, Y}^{*}$.

If $\alpha \in\left(\frac{1}{2}, 1\right)$ the function $g_{x, y}(t)$ is concave and the inequality is reversed.

Finally, since $X \leq_{i c v} Y$ if and only if there exists a $Z$ such that $X \leq_{s t} Z \leq_{c v} Y$ (see for example Theorem 1.5.14 in Muller and Stoyan, 2002), item c) follows immediately from a) and b).

Remark 4 If $\alpha=\frac{1}{2}$, since $x_{\frac{1}{2}, X}^{*}=E[X]$, it follows that $X \leq_{c v} Y \Longrightarrow x_{\frac{1}{2}, X}^{*}=$ $x_{\frac{1}{2}, Y}^{*}$.

Remark 5 In the case of the usual quantiles, the function $g_{x, y}$ in (21) would be replaced by

$$
\widetilde{g}_{x, y}(t):=\left\{\begin{array}{c}
(1-\alpha)(y-x) \text { if } t \leq x \\
(1-\alpha)(y-t)-\alpha(x-t) \text { if } x \leq t \leq y \\
\alpha(x-y) \text { if } t>y
\end{array}\right.
$$

that is decreasing but neither convex nor concave for any value of $\alpha$; for this reason the usual quantiles are isotonic with respect to the $\leq_{\text {st }}$ order but not to the $\leq_{i c v}$ order.

\section{$3 \quad L^{p}$-quantiles}

Chen (1996) generalized the expectiles introducing the notion of $L^{p}$-quantiles, defined as the minimizers

$$
x_{p, \alpha, X}^{*}:=\arg \min _{x \in R} \pi_{\alpha}(X, x)
$$

with

$$
\pi_{\alpha}(X, x)=E\left[\alpha\left((X-x)^{+}\right)^{p}+(1-\alpha)\left((X-x)^{-}\right)^{p}\right]
$$


with $E\left[|X|^{p}\right]<+\infty$. Again, we will sometimes drop the unnecessary subscripts. Chen proved the minimizer is unique and is a solution of the first order condition

$$
(1-\alpha) \int_{-\infty}^{x_{p}^{*}}\left(x_{p}^{*}-t\right)^{p-1} d F(t)=\alpha \int_{x_{p}^{*}}^{+\infty}\left(t-x_{p}^{*}\right)^{p-1} d F(t)
$$

that in the case of an integer $p$ can be rewritten as

$$
(1-\alpha) F_{(p)}\left(x_{p}^{*}\right)=\alpha \bar{F}_{(p)}\left(x_{p}^{*}\right)
$$

where $F_{(p)}$ and $\bar{F}_{(p)}$ are defined recursively as follows:

$$
\begin{aligned}
& F_{(p+1)}(x):=\int_{-\infty}^{x} F_{(p)}(t) d t, F_{(1)}(t):=F(t), \\
& \bar{F}_{(p+1)}(x):=\int_{x}^{+\infty} \bar{F}_{(p)}(t) d t, \bar{F}_{(1)}(t):=\bar{F}(t) .
\end{aligned}
$$

As in the case of expectiles, defining

$$
G(x):=\frac{E\left[\left((X-x)^{-}\right)^{p}\right]}{E\left[|X-x|^{p}\right]}=\frac{F_{(p)}(x)}{F_{(p)}(x)+\bar{F}_{(p)}(x)}
$$

the $L^{p}$-quantiles of $X$ coincide with the usual quantiles of the new distribution $G$. Moreover, it is possible to compute

$$
\frac{d x_{p}^{*}}{d \alpha}=\frac{F_{(p)}\left(x_{p}^{*}\right)+\bar{F}_{(p)}\left(x_{p}^{*}\right)}{(1-\alpha) F_{(p-1)}\left(x_{p}^{*}\right)+\alpha \bar{F}_{(p-1)}\left(x_{p}^{*}\right)}
$$

Typically the higher the order $p$, the more concentrated are $L^{p}$-quantiles around the value of $x_{p, \frac{1}{2}, X}^{*}$; however a general comparison result is lacking. It can be easily proven in the following example:

Example 6 If $X$ has a uniform distribution $U(0,1)$, then

$$
\begin{aligned}
\pi_{\alpha}(X, x) & =(1-\alpha) \int_{0}^{x}(x-t)^{p} d t+\alpha \int_{x}^{1}(t-x)^{p} d t \\
& =(1-\alpha) \frac{x^{p+1}}{p+1}+\alpha \frac{(1-x)^{p+1}}{p+1}
\end{aligned}
$$

the first order condition is

$$
(1-\alpha) x_{p}^{* p}-\alpha\left(1-x_{p}^{*}\right)^{p}=0
$$


and the $L^{p}$-quantile is given by

$$
x_{p}^{*}=1-\frac{1}{1+\left(\frac{\alpha}{1-\alpha}\right)^{\frac{1}{p}}} .
$$

Since

$$
\frac{d x_{p}^{*}}{d p}=\frac{-\frac{1}{p^{2}}\left(\frac{\alpha}{1-\alpha}\right)^{\frac{1}{p}} \ln \left(\frac{\alpha}{1-\alpha}\right)}{\left[1+\left(\frac{\alpha}{1-\alpha}\right)^{\frac{1}{p}}\right]^{2}}
$$

it follows that $x_{p}^{*}$ is an increasing function of $p$ for $\alpha<\frac{1}{2}$, and decreasing for $\alpha>\frac{1}{2}$.

\subsection{Isotonicity properties}

With the same techniques of the preceding section we prove the isotonicity of $L^{p}$-quantiles with respect to the so-called $s$-convex order. We recall that $X \leq_{s-c x} Y$ if and only if $E[f(X)] \leq E[f(Y)]$ for each $s$-convex $f$ such that both integrals are well defined. A function $f: R \rightarrow R$ is $s$-convex if and only if

$$
\Delta^{\varepsilon_{1}} \ldots \Delta^{\varepsilon_{s}}[f(x)] \geq 0
$$

for each $\varepsilon_{1}, \ldots, \varepsilon_{s} \geq 0$, where the difference operator $\Delta^{\varepsilon}$ is defined as

$$
\Delta^{\varepsilon}(f)=f(x+\varepsilon)-f(x) .
$$

The notion of $s$-convexity generalizes the usual notion of convexity; $f$ is 2-convex if and only if it is convex, while $f$ is 1-convex if and only if it is increasing. If $f$ is $s$-times differentiable and $f^{(s)}(x) \geq 0$, then $f$ is $s$-convex; in particular polynomials up to order $s-1$ are $s$-convex, so $X \leq_{s-c x} Y \Longrightarrow E\left[X^{k}\right]=E\left[Y^{k}\right]$ for $k=1, \ldots, s-1$. For further properties of the $s$-convex orders see for example Muller and Stoyan (2002) and the references therein.

Theorem 7 Let $x_{p, \alpha, X}^{*}$ be defined as in (22). We have the following:

a) If $p$ is odd, then $X \leq_{s-c x} Y$ implies $x_{\alpha, X}^{*} \leq x_{\alpha, y}^{*}$ for each $\alpha$; in particular $X \leq_{s t} Y \Longrightarrow x_{\alpha, X}^{*} \leq x_{\alpha, Y}^{*}$

b) If $p$ is even and $\alpha \in\left(0, \frac{1}{2}\right)$, then $X \leq_{s-c x} Y$ implies $x_{\alpha, X}^{*} \geq x_{\alpha, y}^{*}$

c) If $p$ is even and $\alpha \in\left(\frac{1}{2}, 1\right)$, then $X \leq_{s-c x} Y$ implies $x_{\alpha, X}^{*} \leq x_{\alpha, y}^{*}$

Proof. We apply the same technique of Theorem (3). We have that

$$
\pi_{\alpha}(X, y)-\pi_{\alpha}(X, x)-\pi_{\alpha}(Y, y)+\pi_{\alpha}(Y, x)=E\left[g_{x, y}(X)\right]-E\left[g_{x, y}(Y)\right]
$$


with

$$
g_{x, y}(t):=\left\{\begin{array}{c}
\left.(1-\alpha)\left\{(y-t)^{p}-(x-t)^{p}\right\}\right) \text { if } t \leq x \\
(1-\alpha)(y-t)^{p}-\alpha(t-x)^{p} \text { if } x \leq t \leq y \\
\alpha\left((t-y)^{p}-(t-x)^{p}\right) \text { if } t>y
\end{array}\right.
$$

and by a straightforward computation

$$
g_{x, y}^{(p)}(t):=\left\{\begin{array}{c}
0 \text { if } t<x \\
p !\left[(-1)^{p}(1-\alpha)-\alpha\right] \text { if } x<t<y \\
0 \text { if } t>y
\end{array}\right.
$$

If $p$ is odd, then $g_{x, y}^{(p)}=-1$ and hence $X \leq_{s-c x} Y$ implies that $E\left[g_{x, y}(X)\right] \geq$ $E\left[g_{x, y}(Y)\right]$, that gives condition (17), from which a) follows. If $p$ is even and $\alpha \in\left(\frac{1}{2}, 1\right)$, then again $g_{x, y}^{(p)}<0$, and an identical argument proves c). Finally, if $p$ is even and $\alpha \in\left(0, \frac{1}{2}\right)$, then $g_{x, y}^{(p)}>0$, that gives the supermodularity of $\pi_{\alpha}(X, x)$, from which we get $\left.\mathrm{b}\right)$.

\section{$4 \quad M$-quantiles}

$M$-quantiles have been introduced in Breckling and Chambers (1988) as the minimizers

$$
x_{\varphi, \alpha, X}^{*}:=\arg \min _{x \in R} \pi_{\alpha}(X, x)
$$

of the general asymmetric loss

$$
\pi_{\alpha}(X, x)=E\left[\alpha \varphi\left((X-x)^{+}\right)+(1-\alpha) \varphi\left((X-x)^{-}\right)\right]
$$

with $\varphi:[0,+\infty) \rightarrow[0,+\infty)$ increasing and convex. In order to guarantee the finiteness of $\pi_{\alpha}(X, x)$, it is natural to require that $X \in M^{\varphi}$, the so-called Orlicz heart associated to the function $\varphi$, defined as follows:

$$
M^{\varphi}:=\{X: E[\varphi(\lambda X)]<+\infty \text { for each } \lambda>0\}
$$

$M^{\varphi}$ is a Banach space that is a subspace of the Orlicz space $L^{\varphi}$, defined as

$$
L^{\varphi}:=\{X: E[\varphi(\lambda X)]<+\infty \text { for some } \lambda>0\} ;
$$

see Kosmol and Muller-Wichards (2011) for further references on these spaces. The first order conditions for $M$-quantiles can be derived as in the case of Orlicz quantiles introduced in Bellini and Rosazza-Gianin (2011).

Since $\pi_{\alpha}(X, x)$ is convex in $x$ but in general not differentiable, the first order condition will be of the form $0 \in \partial \pi_{\alpha}\left(X, x^{*}\right)$; we can state the following (see Proposition 6 in Bellini and Rosazza-Gianin (2011)): 
Proposition 8 Let $\varphi$ be differentiable and $X \in M^{\varphi}$. Then $x_{\varphi, \alpha, X}^{*}$ in (34) satisfies the following conditions:

$$
\left\{\begin{array}{l}
(1-\alpha) E\left[\mathbf{1}_{\left\{X<x^{*}\right\}}^{\prime} \varphi^{\prime}\left(\left(X-x^{*}\right)^{-}\right)\right]-\alpha E\left[\begin{array}{l}
\mathbf{1}_{\left\{X \geq x^{*}\right\}}^{\prime} \varphi^{\prime}\left(\left(X-x^{*}\right)^{+}\right) \\
(1-\alpha) E\left[\mathbf{1}_{\left\{X \leq x^{*}\right\}}^{\prime} \varphi^{\prime}\right.
\end{array}\left(\left(X-x^{*}\right)^{-}\right)\right]-\alpha E\left[\mathbf{1}_{\left\{X>x^{*}\right\}}^{\prime} \varphi^{\prime}\left(\left(X-x^{*}\right)^{+}\right)\right] \geq 0
\end{array}\right.
$$

For example in the case $\varphi(x)=x$ that corresponds to the usual quantiles we get

$$
\left\{\begin{array}{l}
(1-\alpha) P\left\{X<x^{*}\right\}-\alpha P\left\{X \geq x^{*}\right\} \leq 0 \\
(1-\alpha) P\left\{X \leq x^{*}\right\}-\alpha P\left\{X>x^{*}\right\} \geq 0
\end{array}\right.
$$

that gives

$$
P\left\{X<x^{*}\right\} \leq \alpha \leq P\left\{X \leq x^{*}\right\} .
$$

Remark 9 When $X$ has a continuous distribution or when $\varphi^{\prime}(0)=0$, then $\pi_{\alpha}(X, x)$ is differentiable and the first order condition (36) becomes simply

$$
\alpha E\left[\varphi^{\prime}\left(\left(X-x^{*}\right)^{+}\right)\right]=(1-\alpha) E\left[\varphi^{\prime}\left(\left(X-x^{*}\right)^{-}\right)\right] .
$$

of which (24) and (8) are special cases.

\subsection{Isotonicity results}

In the preceding sections we employed sufficient conditions for increasing optimal solutions in order to prove isotonicity of generalized quantiles with respect to several stochastic ordering. In this section we show that for a general $M$ quantile, that is for a general $\varphi$ in (35), only isotonicity with respect to the first order stochastic dominance holds. To show this, we will apply the necessary conditions for increasing optimal solutions developed in Milgrom and Shannon (1984). In their general setting, Milgrom and Shannon showed that a necessary condition for increasing optimal solutions is that quasisubmodularity of the function $\pi_{\alpha}(X, x)$, that in our case is equivalent to single crossing property of $\pi_{\alpha}(X, x)$, defined as follows: for each $x \leq y$ and $X \precsim Y$,

$$
\begin{aligned}
& \text { i) } \pi_{\alpha}(Y, y)-\pi_{\alpha}(Y, x)>0 \Longrightarrow \pi_{\alpha}(X, y)-\pi_{\alpha}(X, x)>0 \\
& \text { ii) } \pi_{\alpha}(Y, y)-\pi_{\alpha}(Y, x) \geq 0 \Longrightarrow \pi_{\alpha}(X, y)-\pi_{\alpha}(X, x) \geq 0
\end{aligned}
$$

We can then prove the following result: 
Theorem 10 Let $\varphi \in C^{2}[0,+\infty)$, increasing and convex. Let $X \in M^{\varphi}$, and $x^{*}$ as in (34). Then

i) $x^{*}$ is isotone with respect to the $\leq_{s t}$ order

ii) $x^{*}$ is isotone with respect to the $\leq_{c v}$ order if and only if $\varphi^{\prime \prime}(x)=k$, with $k>0$.

Proof. Let $y>x$. As before, we can write

$$
\pi_{\alpha}(X, y)-\pi_{\alpha}(X, x)=E\left[g_{x, y}(X)\right],
$$

with

$$
g_{x, y}(t):=\left\{\begin{array}{c}
(1-\alpha)(\varphi(y-t)-\varphi(x-t)) \text { if } t \leq x \\
(1-\alpha) \varphi(y-t)-\alpha \varphi(t-x) \text { if } x<t \leq y \\
\alpha(\varphi(t-y)-\varphi(t-x)) \text { if } t>y
\end{array}\right.
$$

Since $\varphi \in C^{2}[0,+\infty)$, it follows that $g_{x, y} \in C(R)$ and $g_{x, y} \in C^{2}(R-\{x, y\})$. Moreover,

$$
g_{x, y}^{\prime}(t):=\left\{\begin{array}{c}
(1-\alpha)\left(-\varphi^{\prime}(y-t)+\varphi^{\prime}(x-t)\right) \text { if } t<x \\
-(1-\alpha) \varphi^{\prime}(y-t)-\alpha \varphi^{\prime}(t-x) \text { if } x<t<y \\
\alpha\left(\varphi^{\prime}(t-y)-\varphi^{\prime}(t-x)\right) \text { if } t>y
\end{array}\right.
$$

From the convexity of $\varphi$ it follows that $\varphi^{\prime}$ is increasing, and hence $g_{x, y}^{\prime}(t) \leq 0$; it follows that $g_{x, y}$ is decreasing, that implies i) as before.

From Theorem 4 in Milgrom and Shannon (1984), the single crossing property (38) is a necessary condition for the isotonicity of $x^{*}$ with respect to the $\leq_{c v}$ order. We now show that the convexity of $g_{x, y}$ is a necessary condition for (38). Suppose indeed that $g_{x, y}$ is not convex; then there exist $z_{1}, z_{2}$ and $\alpha \in(0,1)$ such that

$$
g_{x, y}\left(\alpha z_{1}+(1-\alpha) z_{2}\right)>\alpha g_{x, y}\left(z_{1}\right)+(1-\alpha) g_{x, y}\left(z_{2}\right) .
$$

Considering the random variables

$$
X:=\left\{\begin{array}{c}
z_{1} \text { with prob. } \alpha \\
z_{2} \text { with prob. } 1-\alpha
\end{array}\right.
$$

and

$$
Y:=\alpha z_{1}+(1-\alpha) z_{2}=E[X]
$$

we have clearly that $X \leq_{c v} Y$ and $E\left[g_{x, y}(Y)\right]>E\left[g_{x, y}(X)\right]$. With a proper translation of $\varphi$, we can always get $E\left[g_{x, y}(Y)\right]>0$ and $E\left[g_{x, y}(X)\right]<0$, that would give $\pi_{\alpha}(Y, y)-\pi_{\alpha}(Y, x)>0$ and $\pi_{\alpha}(X, y)-\pi_{\alpha}(X, x)<0$, contradicting i) in (38).

By a straightforward computation, we get

$$
g_{x, y}^{\prime \prime}(t)=\left\{\begin{array}{c}
(1-\alpha)\left(\varphi^{\prime \prime}(y-t)-\varphi^{\prime \prime}(x-t)\right) \text { if } t<x \\
(1-\alpha) \varphi^{\prime \prime}(y-t)-\alpha \varphi^{\prime \prime}(t-x) \text { if } x<t<y \\
\alpha\left(\varphi^{\prime \prime}(t-y)-\varphi^{\prime \prime}(t-x)\right) \text { if } t>y
\end{array}\right.
$$


For the convexity of $g$ we need $\varphi^{\prime \prime}(y-t)-\varphi^{\prime \prime}(x-t) \geq 0$ for each $t \leq x \leq y$ and $\varphi^{\prime \prime}(t-y)-\varphi^{\prime \prime}(t-x) \geq 0$ for each $x \leq y \leq t$; this is possible only if $\varphi^{\prime \prime}$ is a constant.

\section{References}

Bellini, F., Rosazza Gianin, E. (2011) "Haezendonck-Goovaerts risk measures and Orlicz quantiles", to appear in Insurance: Mathematics and Economics Breckling, J., Chambers, R. (1988) "M-quantiles", Biometrika, 75, 4, pp. 76171

Chen,Z. (1996) "Conditional $L_{p}$-quantiles and their application to testing of symmetry in non-parametric regression", Statistics and Probability Letters, 29, pp. $107-115$

Ferguson, T.S. (1967) "Mathematical Statistics: a decision theoretic approach" Academic Press

Newey, K., Powell, J. (1986) "Asymmetric least squares estimation and testing", Econometrica 55, pp. 819-47

Koenker, R. (1992) "When are expectiles percentiles" Econometric Theory 8 pp. $526-527$

Koenker, R. (2005) "Quantile regression" Cambridge University Press

Kosmol, P., Muller-Wichards, D. (2011) "Optimization in Function Spaces" De Gruyter

Milgrom, P., Shannon, C. (1994) "Monotone Comparative Statics" Econometrica, vol. 62 , no 1 pp. $157-180$

Muller, A., Stoyan, D. "Comparison Methods for Stochastic Models and Risks", Wiley

Muller, A. "Expectiles as risk measures", presentation at Dagstat conference 2010

Jones (1994) "Expectiles and M-quantiles are quantiles" Statistics and Probability Letters 20, pp. 149-153

Rockafellar, R. T., Uryasev, S. (2002) "Conditional value-at-risk for general loss distributions", Journal of Banking and Finance vol. 26, 7, pp. 1443-1471

Topkis, D.M. (1978) "Minimizing a submodular function on a lattice" Operations Research vol. 26, no. 2, pp. 305-321

Topkis, D.M. (1998) "Supermodularity and complementarity" Princeton University Press 